\title{
Determination of the Aerodynamic Characteristics of Flying Vehicles Using Method Large Eddy Simulation with Software ANSYS
}

\author{
Nguyen Quang Vinh \\ Department of Radio-Electronic, Institute of Science and Technology, Cau Giay District, Hanoi, Vietnam \\ Email address: \\ Vinhquang2808@yahoo.com
}

\section{To cite this article:}

Nguyen Quang Vinh. Determination of the Aerodynamic Characteristics of Flying Vehicles Using Method Large Eddy Simulation with Software ANSYS. Automation, Control and Intelligent Systems. Vol. 3, No. 6, 2015, pp. 118-123. doi: 10.11648/j.acis.20150306.14

\begin{abstract}
Nowadays, together with the huge development in information technology, the use of numerical method is no longer an obstacle. It provides a lot of advantages such as providing high precise solutions, reducing time and cost for designing and manufacturing flying vehicle models and dealing with extremely complex problems by using physical models compared to the usual analytical methods. The operation of airplanes represents a critical aerodynamic practical problem due to the wing. Interference this article presents a method to identify the aerodynamics coefficients of flying vehicles for isolate wing using software ANSYS. CFX and compares obtained results to the with those received by analytical method.
\end{abstract}

Keywords: Aerodynamic Characteristics, Wing, Airfoil Section, Angle of Attack

\section{Introduction}

One of the most practical problems in flight structure design and operation, is the wing ground interference. The aerodynamics characteristics of wings are changed in collision phenomena. In the present paper, the isolate wing is investigated analyzability and numerically. The investigation takes into account the solution of flow around a isolate wing which has profile represented in Fig.1; and a simulation is carried out at 20 angle of attack (AOA). Experimental results are presented for a Mach number ranged from 0.6 to 2.0 at averaged Reynolds number of 0.89 .
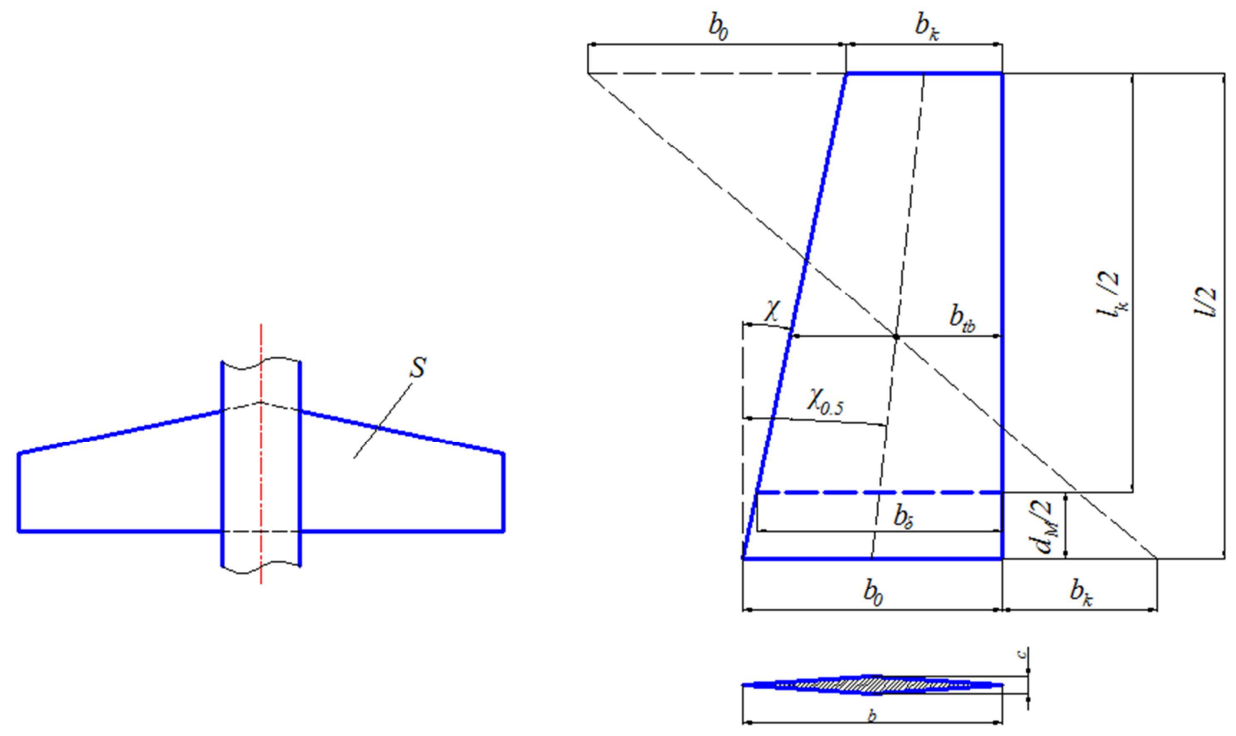

Fig. 1. The model profile of isolate wing for simulating. 
Where $\mathrm{b} 0=1 \mathrm{~m} ; \mathrm{c}=0.064 \mathrm{~mm} ; \mathrm{l}=1.5 \mathrm{~m} ; \mathrm{bk}=0.6 \mathrm{~m}$, the flow around an isolate wing is modeled as incompressible at a Re number. Numerical simulations are performed with the commercial software package ANSYS. CFX and GAMBIT. Simulated results using numerical simulations compared with those using analytical method which is given in [3].

\section{Experimental Procedure}

\subsection{Numerical Simulation Method}

In all efforts aerodynamic studies, no matter whether they are theoretical, experimental or computational are normally aimed at a common objective: to determine the aerodynamic forces and moments acting on a body moving through air. The main purpose of employing Computer Fluid Dynamics (CFD) is to predict the aerodynamic forces: lift and drag, and the pitching moments, acting on the wing, in a way that allows to reduce time and cost when comparing with the real experiment tests.

An algorithm for modeling isolate wing with the profile shown in Fig. 1 is described on schemata Fig. 2 below.

Within the scope of this problem, we will isolate with the following assumptions:

- The surface is smooth channel (bypass roughness, surface profile);

- Wings endothermic (heat exchanger bypass between wing panels and the environment);

- The flow of air to flow under small angle of attack with different velocities corresponding Mach number $\mathrm{M}=0.5$, $0.6,0.7,0.8,0.9,1.0,1.1,1.2,1.3,1.4,1.5,2.0,2.5,3$.

- The nature of the airflow to be taken according to GOST 4401-81: temperature $T_{\infty}=298.15 K$; Pressure $p_{\infty}=101330 \mathrm{~Pa} \quad ; \quad$ dynamic viscosity $v_{\infty}=1.46 \cdot 10^{-5} \mathrm{~m}^{2} / \mathrm{s} ;$ density $\rho_{\infty}=1.225 \mathrm{~kg} / \mathrm{m}^{3}$.

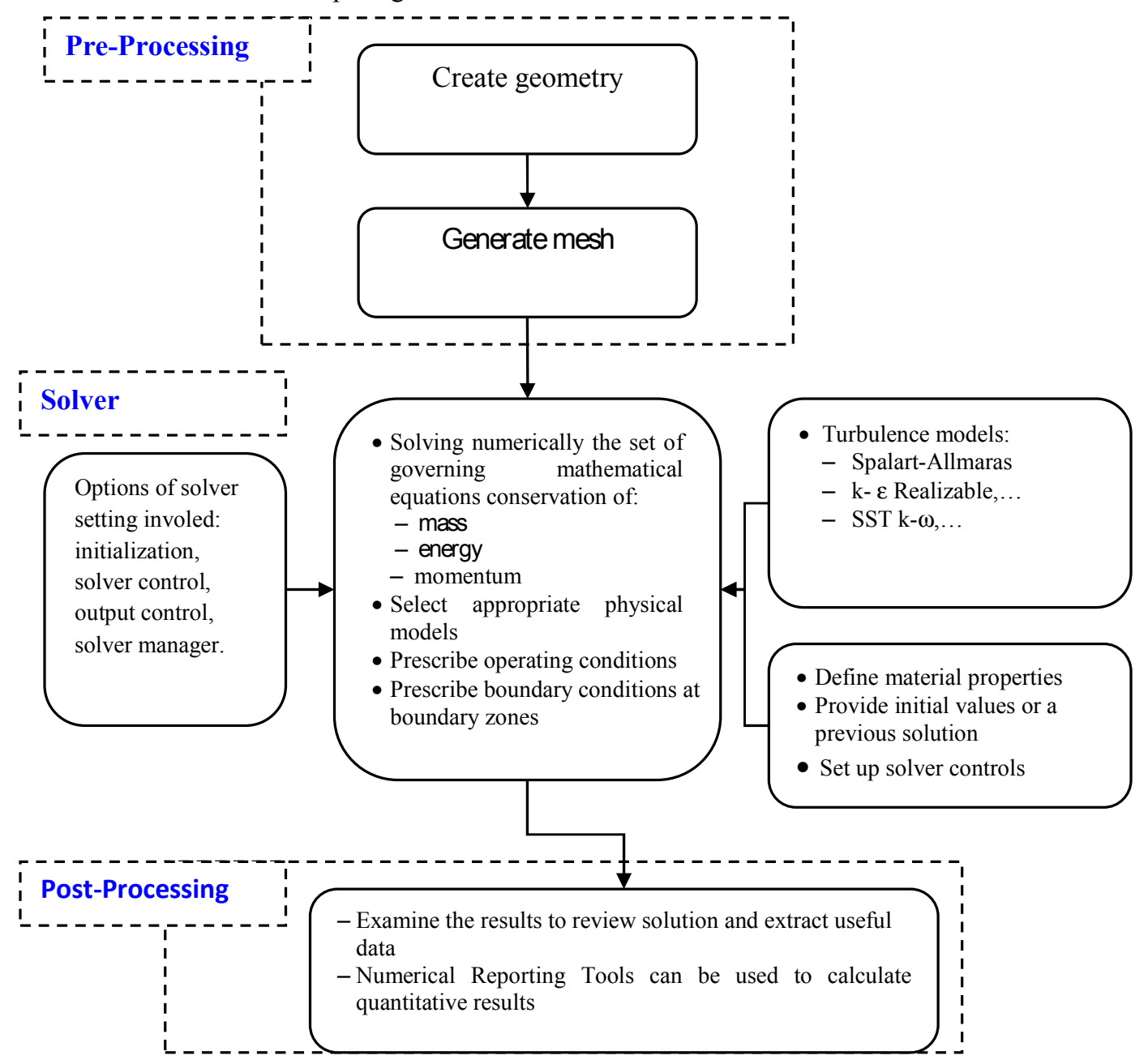

Fig. 2. Scheme of process to simulate considered model.

\subsection{Boundary and Initial Conditions}

In this investigation, considered isolate wing is supposed to have:

- Smooth surface (ignoring the surface roughness).
- Isothermal wing (excepted for heat transfer between plane and environment)

- The flow around wing carried out at 20 AOA and the free stream witch Mach number ranged from 0.6 to 2.0 
at an averaged Reynolds number of 5.0e5-5.2e5.

- The wind tunnel operates at approximately $L \times H \times W=\left(10 b_{0} \div 15 b_{0}\right) \times\left(6 b_{0} \div 8 b_{0}\right) \times\left(6 b_{0} \div 10 b_{0}\right)$ for subsonic and $L \times H \times W=\left(20 b_{0} \div 70 b_{0}\right) \times\left(10 b_{0} \div 30 b_{0}\right) \times\left(10 b_{0} \div 30 b_{0}\right)$ for supersonic

\subsection{Turbulent Model}

Within the scope of this problem, we use the equation of state based on the ideal gas model. Then:

For incompressible gas flow:

$$
\begin{aligned}
& \rho=\rho_{\text {spec }}=\text { const } ; d h=c_{p} d T+\frac{1}{\rho} d p ; \\
& c_{p}=c_{p}(T) ; c_{p}-c_{v}=R
\end{aligned}
$$

or compressed air lines are:

$$
\rho=\frac{p_{a b s}}{R T} ; d h=c_{p} d T ; c_{p}=c_{p}(T) ; c_{p}-c_{v}=R
$$

When conducting the calculation, we can choose the expression depends on the temperature of the heat capacity at constant pressure, $c_{p}$ according to the methods already available to support the following:

- Perfect Calorically $c_{p}=$ const

- Multi-standard mode or Zero Pressure NASA Format [1].

For a three dimensional, steady, incompressible, and turbulent viscous flow, the mass and momentum equations could be written in the Reynolds-Averaged Navier-Stokes:

Continuity equation: $\frac{\partial \rho}{\partial t}+\frac{\partial}{\partial x_{j}}\left(\rho U_{i}\right)=0$

Momentum conservation equation:

$$
\begin{aligned}
& \frac{\partial\left(\rho u_{i}\right)}{\partial t}+\frac{\partial}{\partial x_{j}}\left(\rho u_{i} u_{j}\right)= \\
& =-\frac{\partial p^{\prime}}{\partial x_{i}}+\frac{\partial}{\partial x_{j}} \cdot\left\{\left(\tau_{i j}-\left\langle\rho \overline{u_{i} u_{j}}\right\rangle\right)\right\}+S_{M}
\end{aligned}
$$

Conservation equation of total energy

$$
\begin{aligned}
& \frac{\partial\left(\rho h_{t o t}\right)}{\partial t}-\frac{\partial p}{\partial t}+\frac{\partial}{\partial x_{j}}\left(\rho u_{j} h_{t o t}\right)= \\
& =\frac{\partial}{\partial x_{j}}\left(\lambda \frac{\partial T}{\partial x_{j}}+\frac{\mu_{t}}{\operatorname{Pr}_{t}} \frac{\partial h}{\partial x_{j}}\right)+\frac{\partial}{\partial x_{j}} \cdot\left(u_{j} \cdot\left(\tau_{i j}-\left\langle\rho \overline{u_{i} u_{j}}\right\rangle\right)\right)+ \\
& +u_{j} \cdot S_{M}+S_{E}
\end{aligned}
$$

Here: $p^{\prime}-$ Pressure has been adjusted, taking into account elements of pressure caused by the turbulent kinetic energy and the effective viscosity:

$$
p^{\prime}=p+\frac{2}{3} \rho k+\frac{2}{3} \mu_{e f f} \nabla \cdot U
$$

$$
\tau_{i j}-\text { stress tensor: } \tau_{i j}=\mu_{e f f}\left(2 S_{i j}-\frac{2}{3} \frac{\partial u_{k}}{\partial x_{k}} \delta_{i j}\right)
$$

$S_{i j}, \delta_{i j}-$ respectively, and strain rate tensor function Kronecker function:

$$
S_{i j}=\frac{1}{2}\left(\frac{\partial u_{i}}{\partial x_{j}}+\frac{\partial u_{j}}{\partial x_{i}}\right) ; \quad \delta_{i j}= \begin{cases}1 & i=j \\ 0 & i \neq j\end{cases}
$$

$\left\langle\rho \overline{u_{i} u_{j}}\right\rangle-$ Reynold stress codes. In theory, the eddy viscosity Reynolds stresses depends on the average velocity gradient and diffusion gradient swung by the following expression:

$$
-\left\langle\rho \overline{u_{i} u_{j}}\right\rangle=2 \mu_{t} S_{i j}-\frac{2}{3}\left(\mu_{t} \frac{\partial u_{k}}{\partial x_{k}}+\rho k\right) \delta_{i j}
$$

$\mu_{\text {eff }}-$ the actual viscosity of the flow. $\mu_{\text {eff }}$ quantity is determined by the formula: $\mu_{\text {eff }}=\mu+\mu_{t}$

$\mu$-viscosity is determined according to the formula on the basis of the model is supported by ANSYS. CFX such as Ideal Mixture, Non-Newtonian Model, Sutherland's Formula ...

$\mu_{t}$ - turbulent viscosity (or viscosity vortex) generated by the turbulence and is determined according to the formula depending on the model of turbulence that we choose.

In the solution of the conservation equations, the turbulence term should be replaced by suitable equivalent model; otherwise, the equations are not solvable. The model used to solve the simulation is SST $k-\omega$. The SST $k-\omega$ turbulence model is a two-equation eddy-viscosity model that is used for many aerodynamic applications. It is a hybrid model combining the Wilcox k-omega and the k-epsilon models.

GAMBIT 2-4.6 and ANSYS. CFX 12.1 are used for modeling and simulation respectively.

\section{Semi-empirical Method}

\subsection{Determine the Lift Coefficient}

When small angle of attack and angle helical wing $\alpha \leq$ $100 ; \delta=0$ twisted wing is the lift coefficient depends on angle of attack is almost linear. Therefore, the lift coefficient can be expressed as follows [5]:

$$
c_{y \text { wing }}=c_{y . \text { wing }}^{\alpha} \alpha=\left(c_{y 1 . \text { wing }}^{\alpha}-\frac{c_{x 0 . w i n g}}{57.3}\right) \alpha
$$

$c_{y 1 \text { wing }}^{\alpha}-$ partial lifting force coefficient $c_{y 1 \text { wing }}$ according to the angle of attack in the coordinate system Oxly1z1. $c_{y 1 \text {.wing }}^{\alpha}$ depending on the number $M_{\infty}$ and shape on plane wing: elongation $\lambda_{\text {wing }}$, spasm of wings $\eta_{\text {wing }}$ and swingwing corner $\chi . \bar{c}$ - relatively thick wing profile, as 
measured in section parallel to the plane wing symmetry of flying machines. The dimensionless quantity is determined according to the following formula:

$$
\bar{c}=\frac{c}{b} ; \quad \eta_{\text {wing }}=\frac{b_{0}}{b_{k}} ; \quad \lambda_{\text {wing }}=\frac{l^{2}}{S}
$$

$c_{y 1 \text {.wing }}^{\alpha}$ - defined by the expression $[4,5]$ :

$$
\frac{c_{y 1 \text { wing }}^{\alpha}}{\lambda_{\text {wing }}}=f\left(\lambda_{\text {wing }} \sqrt{\left|M^{2}-1\right|} ; \lambda_{\text {wing }} \operatorname{tg} \chi_{0,5} ; \lambda_{\text {wing }} \sqrt[3]{\bar{c}}\right)
$$

$c_{x 0 . \text { wing }}-$ coefficient of drag, lift the front as zero

\subsection{Identify the Front Drag Coefficient}

Resistance coefficient frontally $c_{x . w i n g}$ is defined by the expression $[4,5]$ :

$$
c_{x . \text { wing }}=c_{x 0 \text { wing }}+c_{x i \text { wing }} \text {. }
$$

$c_{\text {xi.wing }}$ - induced drag coefficient, which is generated by the lift, which is determined by the formula:

$$
c_{x i \text { wing }}=c_{y . \text { wing }}^{\alpha}\left(K_{\alpha \alpha}-57,3 \xi \bar{c}_{F} c_{y . \text { wing }}^{\alpha} k_{\alpha \alpha}^{2}\right) \frac{\alpha^{2}}{57,3}
$$

$K_{\alpha \alpha}, k_{\alpha \alpha}$ - interference coefficient between the wings and fuselage. In isolated cases wing $K_{\alpha \alpha}=k_{\alpha \alpha}=1$.

$\bar{c}_{F}, \xi-$ its corresponding absorption coefficient and its efficiency coefficient. For wings with sharp edges before then $\bar{c}_{F}=\xi=0$.

$c_{x 0 \text {.wing }}$, code for the next case without jail wing after wing, which is determined by the formula [4.5]: $c_{x 0 \text { wing }}==c_{x p \text {.wing }}+c_{x b \text {.wing }}$

$c_{x p \text {.wing }}-$ coefficient of drag caused by the viscosity of the gas stream to flow. This coefficient is determined by the following formula: $c_{x p \text {.wing }}=(2 f)_{M=0} \eta_{M} \eta_{c}$.

$\eta_{M}, \eta_{c},(2 f)_{M=0}$ is determined according to experimental results in $[4,5]$.

$c_{x b \text { wing }}-\mathrm{A}$ coefficient of drag caused by the compressibility of the flow. The value of this coefficient, depending on the Mach number, the plane wing shape, thickness contour wing and wing shape, which is determined from the expression dependent $[4,5]$ :

$$
\frac{c_{x b . \text { wing }}}{\lambda_{\text {wing }} \bar{c}^{2}}=f\left(\begin{array}{l}
\lambda_{\text {wing }} \sqrt{\left|M^{2}-1\right|} ; \lambda_{\text {wing }} \operatorname{tg} \chi ; \\
\eta_{\text {wing }} ; \text { wing's profine }
\end{array}\right)
$$

Results determined coefficient method and empirical theory is presented in the form of graphs in the document [5].

\section{Results and Discussion}

The simulated results are obtained using numerical simulation with software package ANSYS. CFX and GAMBIT. The Fig. 3 to Fig. 6 are the plots of the pressure contour effort to wing surface with different Mach numbers.

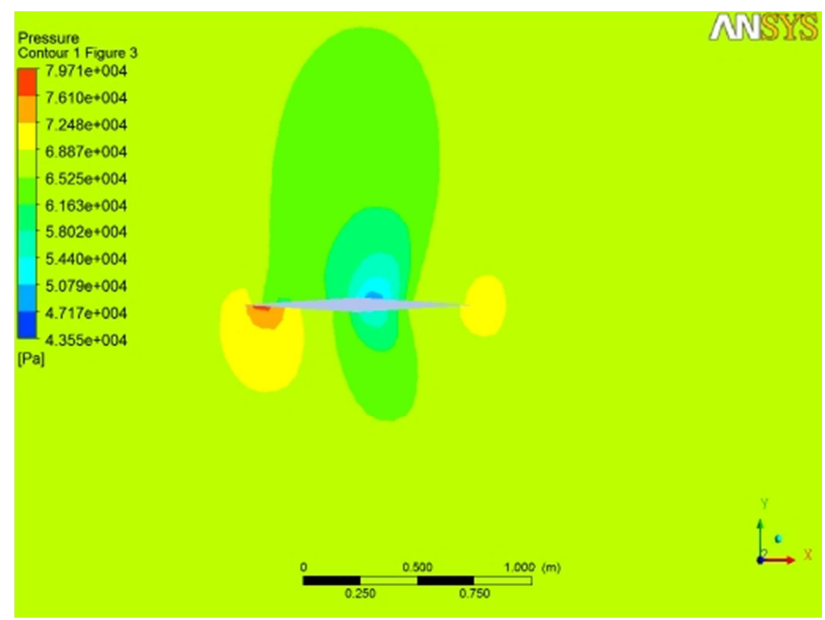

Fig. 3. Pressure contour at $M=0.8$.

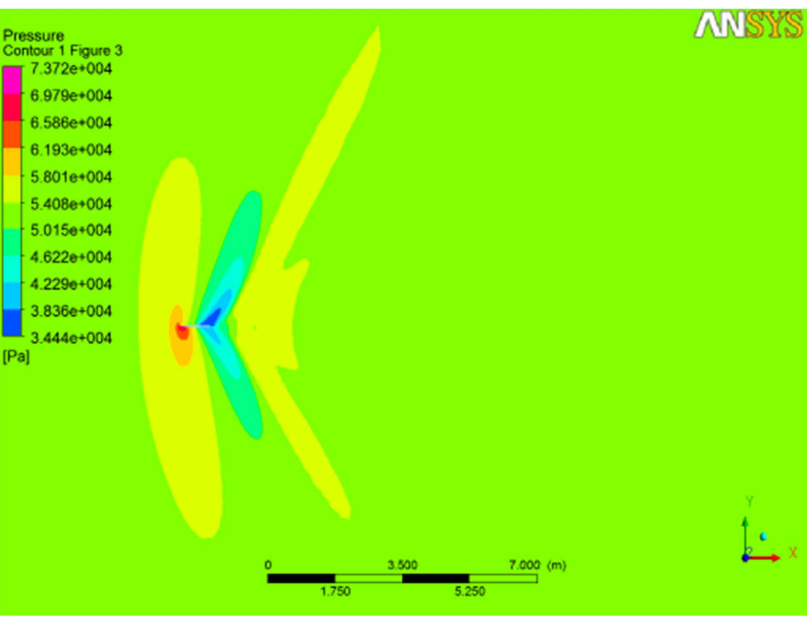

Fig. 4. Pressure contour at $M=1.0$.

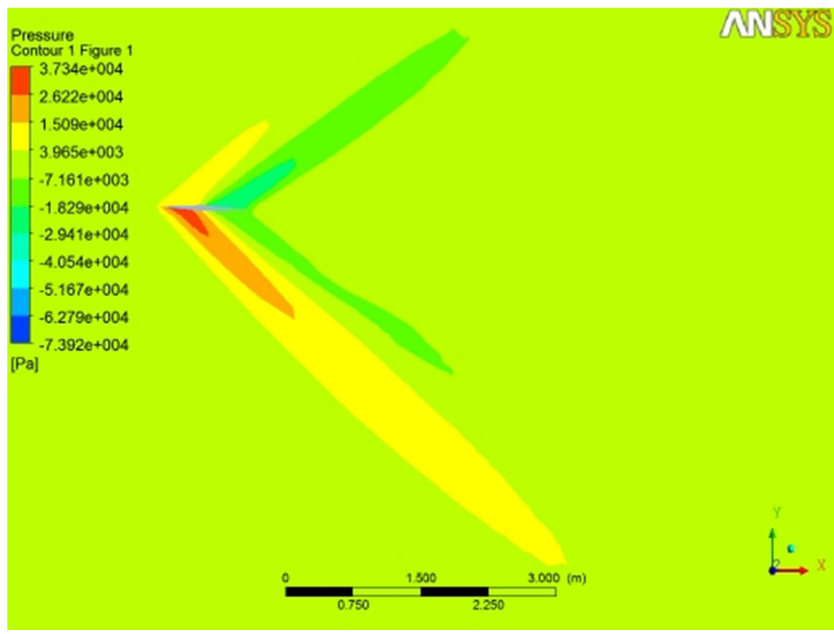

Fig. 5. Pressure contour at $M=1.5$. 


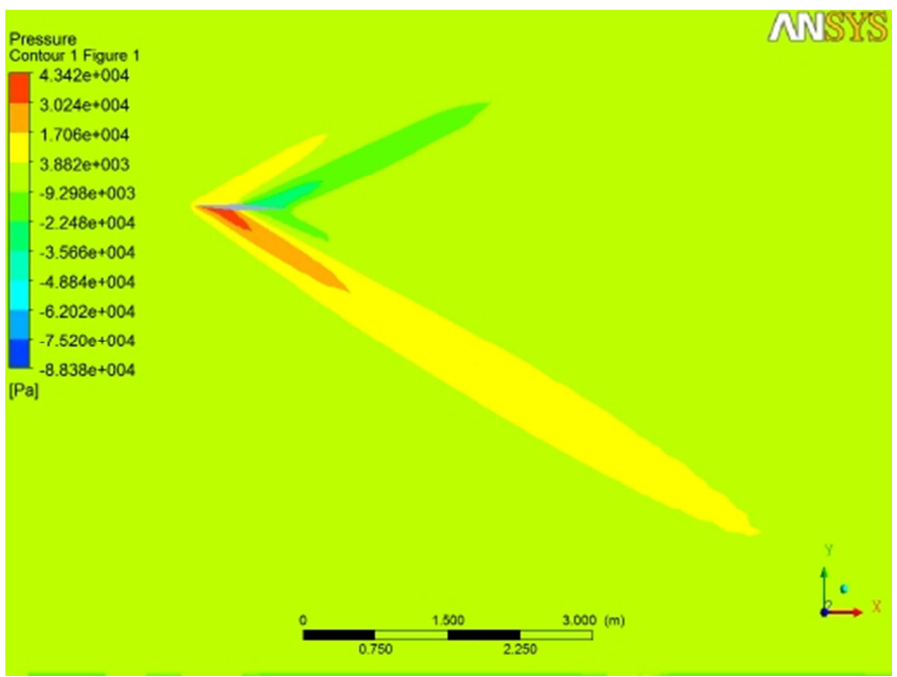

Fig. 6. Pressure contour at $M=2.0$.

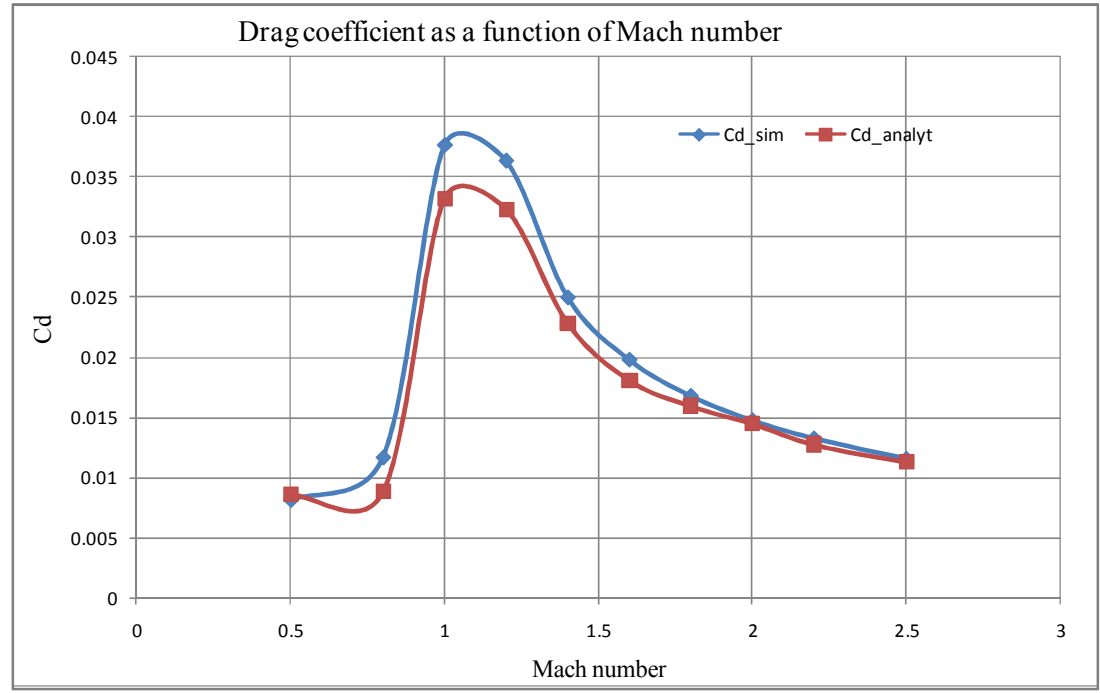

Fig. 7. Variation of drag coefficient as function of $M$.

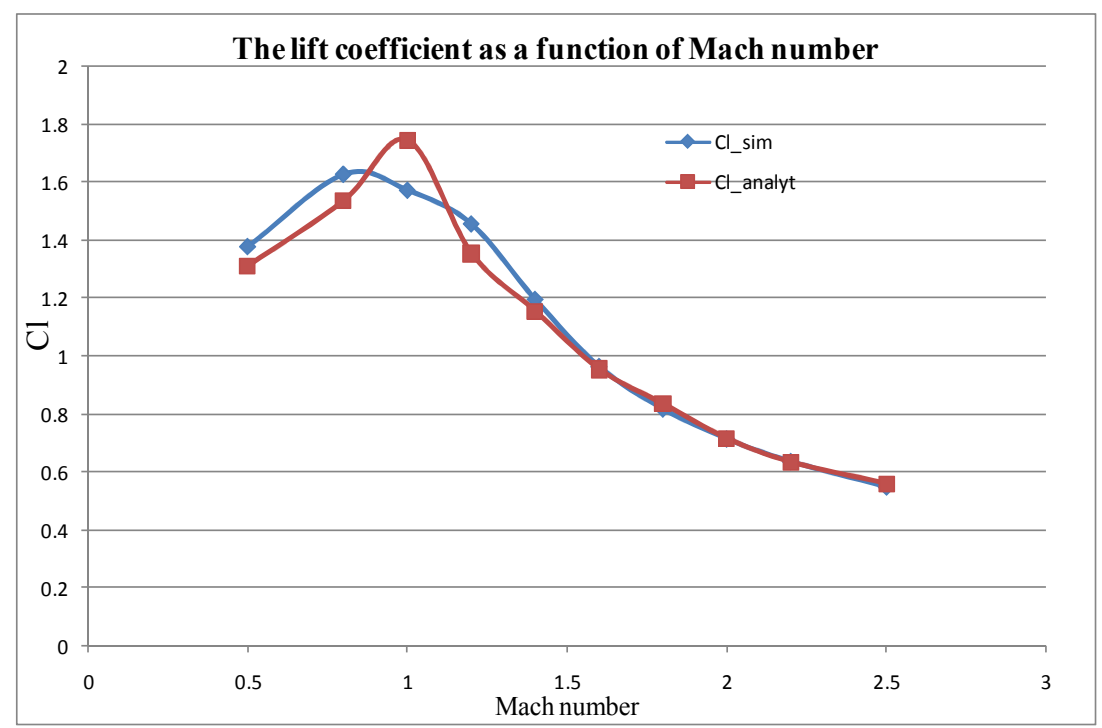

Fig. 8. Variation of drag coefficient as function of $M$. 
In Fig. 3 to Fig. 6 can be observed that the total pressure increases when Mach number increases from 0.5 to about 1.2 , and decreases when the Mach number is over 1.2

Figure 7 are showing the results of the drag, lift coefficients at $20 \mathrm{AOA}$ and the Mach number varies in the range from 0.6 to 2.5 , respectively as obtained from the analytical and numerical simulation. Quite good agreement between the analytical and numerical results could be realized. The variation of the lift coefficient between the analytical and numerical simulation cause the assumption we have represented above (include smooth surface, isothermal wing ...).

\section{Conclusions}

The collision phenomena with influence on the aerodynamic characteristics of isolate wing operation were investigated analytically and numerically. On the numerical part, the conservations of mass and momentum equations are solved by software package ANSYS. CFX using finite volume technique. To close the domain, the turbulence part of the flow is approximated by using SST $k-\omega$ model. The aerodynamic parameters, lift, drag coefficients were measured analytically and predicted numerically at 20 and various Mach number in the range from 0.6 to 2.5 . The results are compared with the analytical method and good agreements is achieved.

\section{Acknowledgements}

The authors would like to thank colleagues in Vietnam Academy of Military Science and Technology for their assistance during the use of ANSYS's laboratory to produce the wing model.

\section{References}

[1] Ansys. CFX Release 12.0: ANSYS CFX-Solver Theory Guide.

[2] Ansys. Gambit Helps.

[3] Zakharchenko VF, et al., Determination of total aerodynamic characteristics of various configurations of aircraft. M: Publishing house of the MSTU. Bauman, 1993.

[4] AA Lebedev, Chernobrovkin LS Flight Dynamics: unmanned aerial vehicles. M: Mechanical Engineering, 1973.

[5] Process and research on the method of guidance a maneuvering target with scholastic prediction about its motion and with nonlinearities in the control circle" (A report on the scientific researching work)/MSTU named after Bauman. The supervisor: Pupkov K. A. The executors: Nguyen Quang Vinh, Bobkov A. V., Ustiuzanin A. D. / Moscow, 2006, 75 pages. 